\section{AUTOLOGOUS STEM CELL TRANSPLANTATION,FOR REFRACTORY COELIAC DISEASE TYPE II: A PROMISING THERAPEUTIC OPTION-THE IRISH EXPERIENCE}

C Kiat, ${ }^{1}$ I Feeley, ${ }^{2}$ Y Y Hong, ${ }^{1}$ P Maheshwari, ${ }^{2}$ C Mulder, ${ }^{3}$ A Hayat, ${ }^{1}$ C Goulding, ${ }^{1}$ V Byrnes' ${ }^{1}$ UHG, Galway, Ireland; ${ }^{2}$ Midwestern Regional Hospital, Limerick, Ireland; ${ }^{3}$ VU University Medical Centre, Amsterdam, The Netherlands

10.1136/gutjnl-2013-305143.76

Introduction Refractory Coeliac disease (RCD II) is a rare but serious complication of celiac disease and is characterized by non-responsiveness to a gluten free diet in the presence of a clonal population of $\mathrm{T}$ lymphocytes within the small intestine. The risk of progression from RCD II to enteropathy-associated T-cell lymphoma (EATL) is estimated at $60-80 \%$ and is associated with a very poor survival.

Aims/Background Therapeutic options for RCD II are limited. Immunosuppressions with corticosteroids, thiopurines and infliximab have been used with disappointing results and may indeed promote the progression to lymphoma. To date, the use of a novel therapeutic approach of high-dose chemotherapy followed by autologous stem cell transplant (ASCT) in a small cohort of patients with RCD II has stimulated great interest world wide with promising results.

Method We describe the clinical presentations and courses of two very different patients with RCD II who received an ASCT. Results Case 1: 36-year-old female with an 8 year history of CD who presented with a weight loss of $8 \mathrm{kgs}$ over the course of 3 months in conjunction with abdominal pain, bloating, and diarrhoea (8-10 times/day). She was fully compliant with a GFD and her anti-tTG was $<1$ units/L. Investigations included a panendoscopy which revealed severe ulcerative jejunitus. Duodenal biopsies revealed loss of CD 8-positive cells and T cell clonality consistent with RCD type II. Abdominal CT revealed abnormal thickening of her jejunum to her mid ileum with mesenteric lymphadenopathy. Extensive investigation for overt lymphoma proved negative. She was unable to maintain adequate nutrition via the enteral route and had a nadir albumin of $7 \mathrm{~g} / \mathrm{L}$. She was commenced on total parenteral nutrition (TPN). She was initially treated with intravenous cortico-steroids followed by high dose chemotherapy in the form of cladribine but failed to respond clinically with ongoing severe malabsorption. In light of this she proceeded to ASCT in July 2012 and in spite of a stormy post transplant course is currently doing well clinically, with resolution of her symptoms and continues to thrive on an oral GFD.

Case 2: 67-year-old female with a one year history of celiac disease on a background of osteoporosis and hypothyroidism. Her symptoms had begun 3 years earlier and failed to improve on a gluten free diet (GFD). At presentation she weighed $37 \mathrm{~kg}$. Coinciding with this she also experienced progressive ataxia which was deemed to be associated with CD. Panendoscopy revealed severe villous atrophy with ulcerative jejunitis. Small bowel biopsies revealed abnormal phenotypic expression of T-lymphocytes with loss of CD 8-positive cells with T-cell clonality consistent with RCD type II. MRI small bowel follow through and PET scan proved negative for overt lymphoma. She was commenced on cortico-steroids and azathioprine (AZA) which was associated with a clinical improvement and a weight gain of 10 $\mathrm{kg}$. However, in spite of this repeat small bowel biopsies confirmed that $>70 \%$ of her $\mathrm{T}$ lymphocytes were monoclonal and her ataxia continued to progress. In light of this she proceeded to ASCT in December 2011. Post transplant she has maintained her weight and her ataxia has remained stable.

Conclusion RCD II is a potentially lethal complication of CD and remains very difficult to treat. In the absence of published randomized clinical trials, evidence is growing for the safety and feasibility of ASCT in this disease.

Since 2004, 24 patients worldwide (including the 2 described here) have received ASCT for this condition and only one patient to date has developed lymphoma (4 years post ASCT). Our 2 patients are the first and only patients in Ireland and the UK to receive an ASCT for RCD II. In the future multicentre randomized trials or trials with historical control groups will be needed to consolidate this treatment but for now we encourage consideration of ASCT for a subgroup of patients with RCD II who would heretofore have a very high mortality risk from sepsis or lymphoma. 